\title{
EAS spectrum in thermal neutrons measured with PRISMA-32
}

\author{
A.A. Petrukhin ${ }^{\star, a}$, D.M. Gromushkin ${ }^{a}$, O.B. Shchegolev ${ }^{b}$, Yu.V. Stenkin ${ }^{a, b}$, \\ V.I. Stepanov ${ }^{b}$, I.I. Yashin ${ }^{a}$ \\ ${ }^{a}$ National Research Nuclear University MEPhI (Moscow Engineering Physics Institute), \\ Moscow 115409, Russian Federation \\ ${ }^{b}$ Institute for Nuclear Research of RAS, Moscow 117312, Russian Federation \\ E-mail: AAPetrukhin@mephi.ru,DMGromushkin@MEPhI.ru
}

Results on EAS study with a novel type detector array PRISMA-32 sensitive to accompanying EAS thermal neutrons are presented. The array is running in MEPhI (Moscow, Russia) since February, 2012. Comparison with Monte Carlo simulations of the experiment using the CORSIKA code is also shown. It is demonstrated that absolute number of recorded thermal neutrons is in a good agreement with expectations and that the EAS size spectrum in thermal neutrons within experimental errors can be well fitted with a pure power law function.

The 34th International Cosmic Ray Conference

30 July - 6 August, 2015

The Hague, The Netherlands

*Speaker 


\section{Introduction}

Hadronic component of extensive air showers (EAS) is very important, but the neutron component has not been practically studied up to now. Absence of effective and inexpensive neutron detectors of large area limited these studies for many years. Developing of such detectors in INR RAS [1], called as en-detectors, allowed one to use them for construction of the novel type EAS array (PRISMA project) [2,3]. The first array of such type PRISMA-32 consisting of 32 en-detectors has been constructed in MEPhI in the frame of MEPhI-INR cooperation. The detectors are sensitive to electromagnetic (e) and neutron (n) EAS components and record thermal neutrons accompanying EAS at a whole area of the array. Details of the array can be found elsewhere [4-6].

EAS size in thermal neutrons was never studied before, and we present here our results obtained after about 3 years of the PRISMA-32 continuous operation.

\section{Method}

The array is deployed inside the NEVOD detector building around the water pool (see Fig. 1). The latter explains its inhomogeneous structure. An efficiency of the en-detector for thermal neutron recording was found to be $20 \%$. The neutrons are recorded as delayed pulses within a time gate of $20 \mathrm{~ms}$. It is more than enough to collect a great bulk of thermal neutrons produced by EAS hadrons [7]. On-line program pre-analyzes the data and stores the energy deposit (above a threshold of 5 m.i.p.) and the number of recorded neutrons in each detector. Two-fold coincidence trigger condition is applied for each cluster of 16 en-detectors to store the data. Additionally, during off-line data processing, 6-fold coincidence (from 32) is used for the event selection.

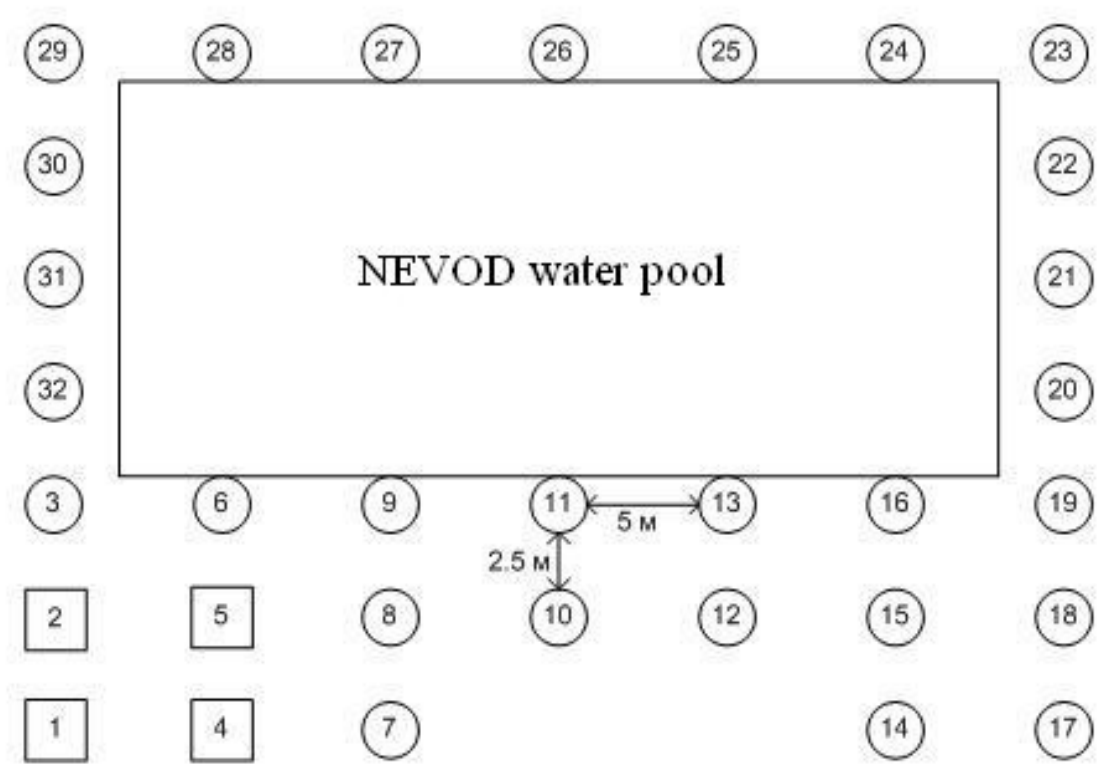

Figure 1: Arrangement of detectors in the array. Detectors 1, 2, 4, and 5 are pyramidal in shape and have the area of $0.75 \mathrm{~m}^{2}$; the remaining detectors are cylindrical and have the area of $0.36 \mathrm{~m}^{2}$. 
We applied a traditional maximum-likelihood analysis of EAS, employing the NishimuraKamata-Greisen (NKG) function for the electromagnetic component to find the position of the EAS axis, its age, EAS size $(\mathrm{Ne})$ and also the total number of recorded neutrons. As it has been shown earlier [8], the total number of secondary neutrons (mostly evaporation) is proportional to the number of hadrons passing through the array area. Therefore, the measuring of the EAS size spectrum in thermal neutrons is equivalent to that in the number of hadrons. Taking into account that hadrons are the main EAS component forming its structure, one could expect that the number of recorded neutrons would provide the most adequate primary energy estimator. Therefore the EAS size spectrum in thermal neutrons can be easily used for primary cosmic ray energy spectrum recalculation.

At this step, CORSIKA-based Monte-Carlo simulations were made only to calculate the relation between the number of recorded neutrons and primary energy.

\section{Results}

EAS size distribution in thermal neutrons measured by PRISMA-32 for 3 years is presented in Fig. 2. As it is mentioned above, a standard procedure was used to locate EAS axis and to estimate other parameters. In addition, a distribution in the number of recorded neutrons was accumulated for events with the axis inside the array area. As one can see, the integral distribution follows a power law with index $\beta=1.95 \pm 0.05$. Primary energies (both for protons and iron) calculated using preliminary CORSIKA simulations are shown at the figure top.

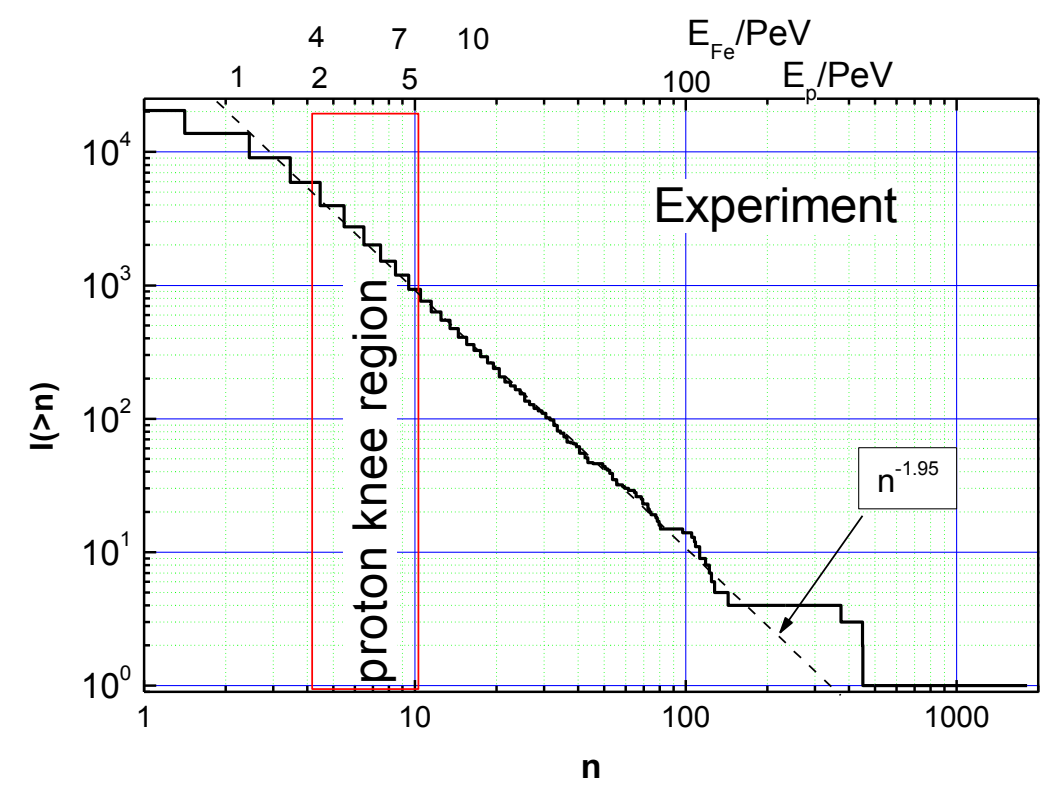

Figure 2: Experimental integral distribution in the number of thermal neutrons.

The same data multiplied by $\mathrm{n}^{1.95}$ are shown in the Fig. 3. It is seen that the power law index for the integral spectrum is very close to 1.95 and is close to our expectations in a case of pure power law primary spectrum with the index of $\gamma=-1.7$. It demonstrates that the EAS size spectrum in thermal neutrons within the experimental errors can be well fitted with a pure power law function [8]. 


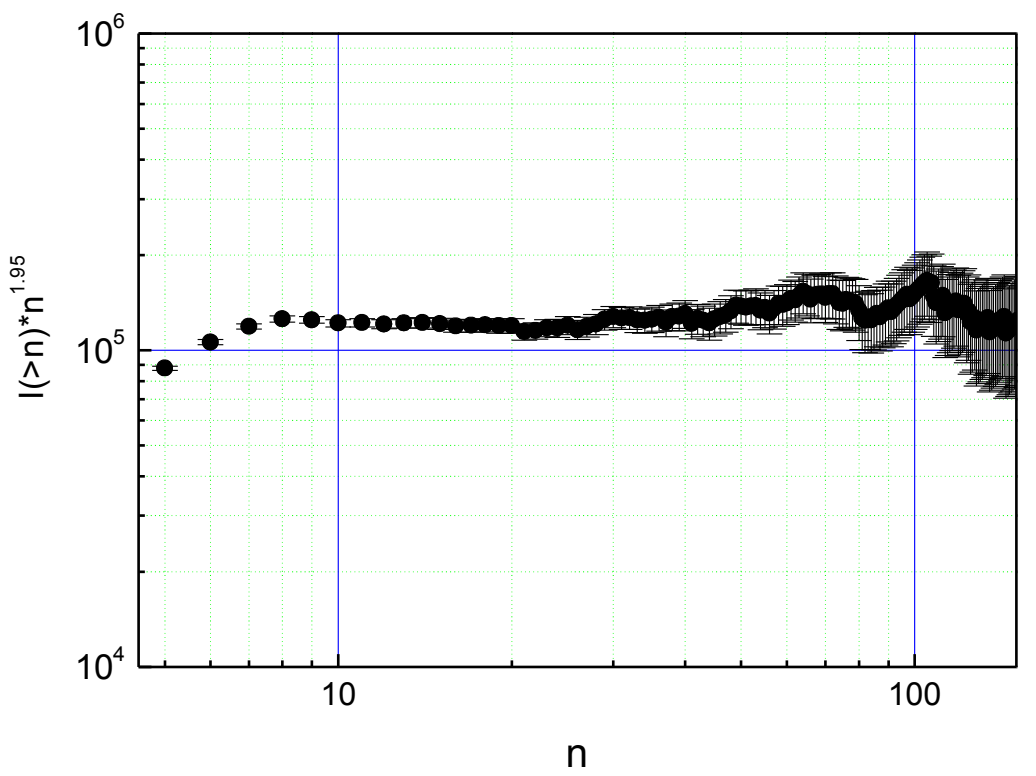

Figure 3: EAS size integral distribution in thermal neutrons multiplied by $n^{1.95}$.

Full-scale simulation based on CORSIKA and GEANT has been performed to obtain correlation between primary energy and recorded number of thermal neutrons in all detectors (Fig. 4). The same program was used to process both simulated and experimental data. The calculations are in progress now and at this stage we show only results with rather poor statistics.

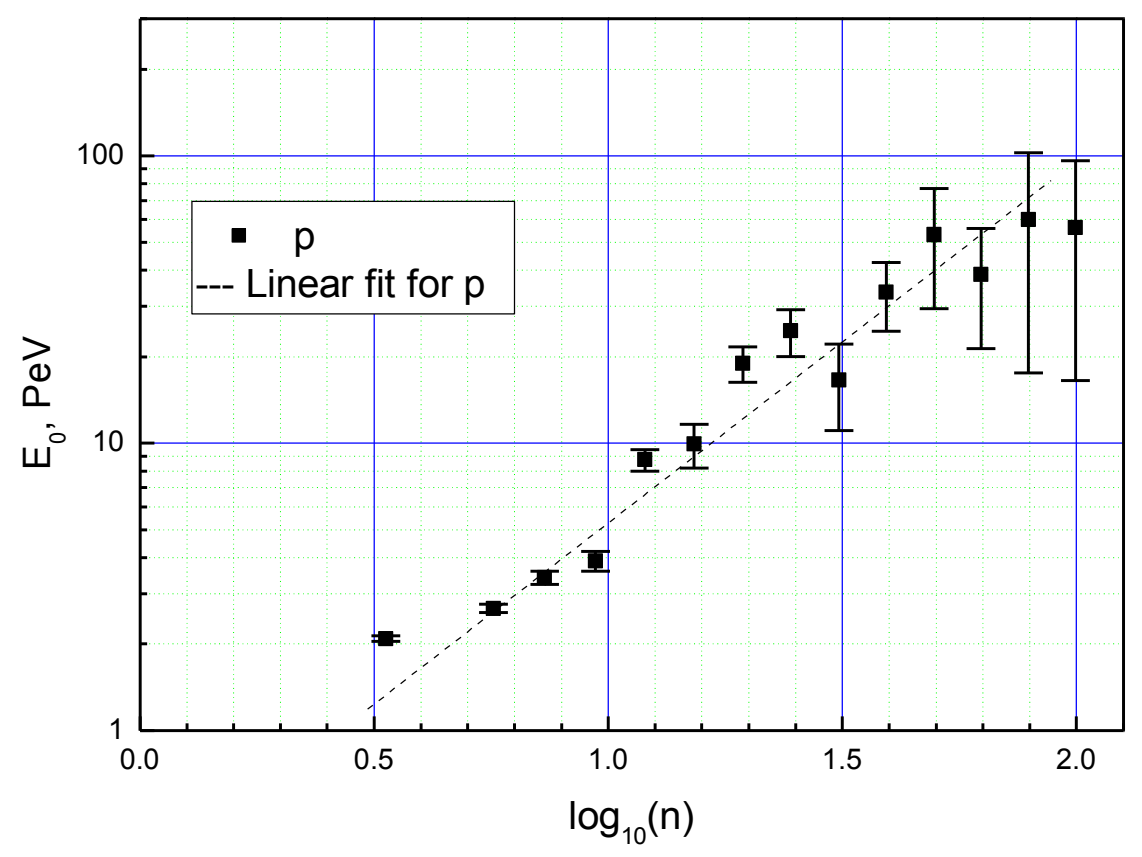

Figure 4: Simulation (CORSIKA + GEANT). 


\section{Conclusion}

A novel type EAS experiment (PRISMA-32) is running in MEPhI (Moscow) for more than 3 years. It is noticeable that for the first time EAS size spectrum in thermal neutrons measured with the array shows a pure power law behavior above the knee region.

\section{Acknowledgements}

This study was performed at the Unique Scientific Facility "Experimental complex NEVOD" in frame of MEPhI and INR RAS cooperation and was supported by the Ministry of Education and Science of the Russian Federation (project no. RFMEFI59114X0002 and no. 4930.2014.2 for support of Leading Scientific Schools), and also funded in part by the Russian Foundation for Basic Research (project 14-02-00996-a) and by the Program of the Presidium of the Russian Academy of Sciences "Fundamental Properties of Matter and Astrophysics".

\section{References}

[1] Yu.V. Sten'kin. Large scintillator detector for thermal neutron recording. In: Nuclear Track Detectors: Design, Methods and Applications ISBN: 978-1-60876-826-4, Editors: M. Sidorov and O. Ivanov, Nova Science Publishers, Inc., Chapter 10, 253 (2010).

[2] Yu.V. Stenkin. Thermal neutrons in EAS: a new dimension in EAS study. Nucl. Phys. B (Proc. Suppl), 175-176, 326 (2008).

[3] Yu.V. Stenkin. On the PRISMA project. Nucl. Phys. B (Proc. Suppl.), 196, 293 (2009).

[4] D. Gromushkin, V. Alekseenko, A. Petrukhin, et al. The array for EAS neutron component detection. JINST, 9, C08028 (2014).

[5] D.M. Gromushkin, V.V. Alekseenko, A.A. Petrukhin, et al. The ProtoPRISMA array for EAS study: first results. Journal of Physics: Conference Series, 409, 012044 (2013) .

[6] D.M. Gromushkin, V.I. Volchenko, A.A. Petrukhin, et al. Novel method for detecting the hadronic component of extensive air showers. Physics of Atomic Nuclei, 78, 349 (2015).

[7] Yu.V. Stenkin, D.M. Gromushkin, A.A. Petrukhin, et al. EAS thermal neutron lateral and temporal distributions. 33 ICRC, Brazil, ID 482 (2013).

[8] Yu.V. Stenkin, V.V. Alekseenko, D.M. Gromushkin, et al. Thermal neutron flux produced by EAS at various altitudes. Chinese Physics C, 37, 015001 (2013). 